
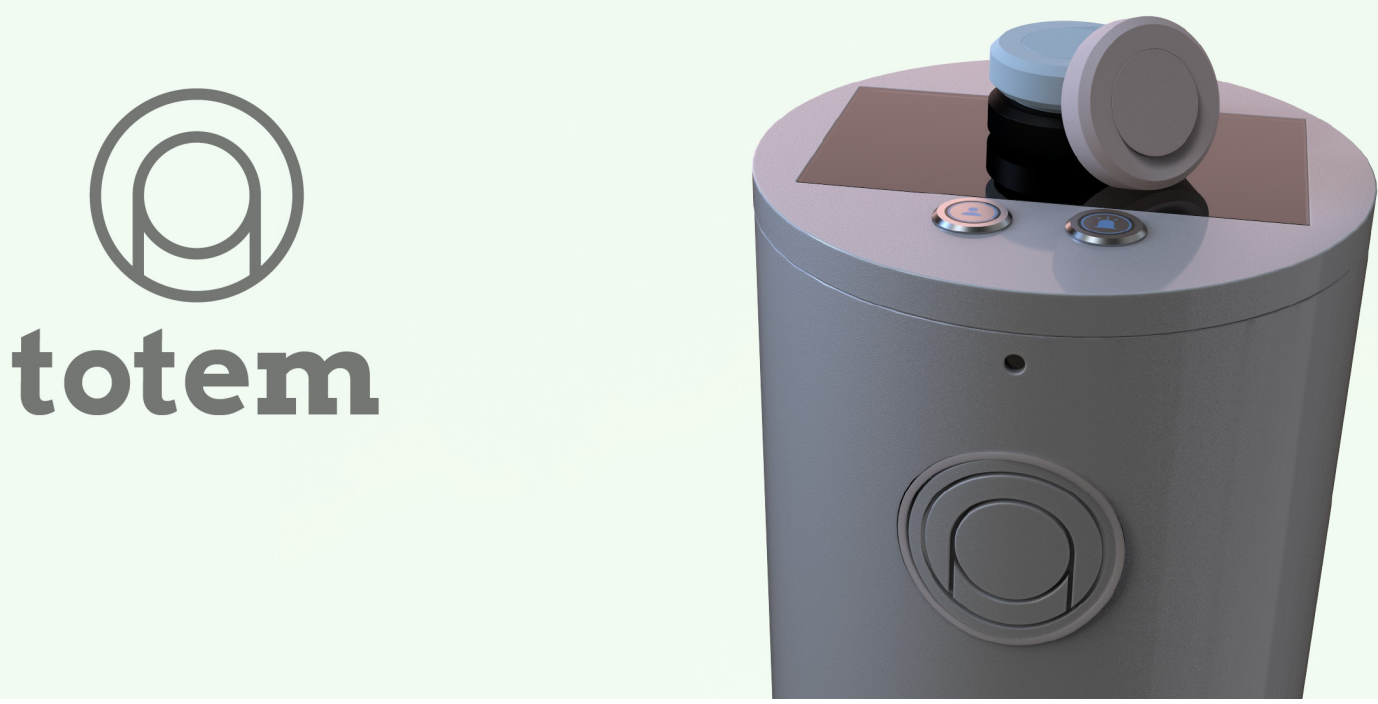

\title{
Diseño piloto de sistema de productos inteligentes para aumentar la sensación de seguridad en las calles de Costa Rica
}

Pilot Smart Product Design to increase the sensation of safeness in the streets of Costa Rica

Mónica I. González-Sosa ${ }^{1}$, Jose P. Corella-Vargas ${ }^{2}$

M.I. González-Sosa, J.P. Corella-Vargas, “Diseño piloto de sistema de productos inteligentes para aumentar la sensación de seguridad en las calles de Costa Rica", IDI+, vol. 3, no 2, Ene., pp. 16-30, 2021.

doi https://www.doi.org/ 10.18845/ridip.v3i2.5563

Fecha de recepción: 5 de agosto de 2020 Fecha de aprobación: 6 de octubre de 2020

1. Mónica I. González-Sosa Estudiante de Ingeniería en Diseño Industrial Instituto Tecnológico de Costa Rica Cartago, Costa Rica 717mgs@gmail.com

(D) 0000-0001-6853-4146

2. Jose P. Corella-Vargas Estudiante de Ingeniería en Diseño Industrial Instituto Tecnológico de Costa Rica Cartago, Costa Rica jpcorella97@gmail.com

(D) 0000-0002-1553-2355 


\section{Resumen}

En el contexto nacional y mundial, se observa muchísima inseguridad en las calles. Desde asaltos hasta secuestros, estos eventos suceden con mucha frecuencia y la mayoría de las veces las personas no tienen cómo alertar o prevenir la situación. Después de meses de ver las mismas noticias en los periódicos, se decidió desarrollar un conjunto de productos inteligentes para mejorar la situación.

Se analizó el mercado para definir la necesidad más importante de todas: poder alertar a los seres queridos y autoridades a la hora de un asalto $u$ otra experiencia similar. A partir de esto, se desarrolló un diseño de producto con la metodología de diseñar el objeto de adentro hacia afuera. Por medio de modelado y simulaciones en 3D, se conceptualizó un conjunto de productos inteligentes para satisfacer la necesidad principal y brindarles a los usuarios una herramienta de apoyo para la situación de la inseguridad. Los productos propuestos pueden generar una nueva sensación al caminar por las calles, además de apoyar el sentido de comunidad en las personas para este tipo de casos, ya que la aplicación funciona de mejor manera si los usuarios del producto le aportan información de las calles.

Aunque el vandalismo en las calles no desaparecerá, es una manera de lograr que las personas se sientan un poco más seguras al transitar por ellas, al ser conscientes de que sus seres queridos pueden saber dónde están y si están bien, en caso de que pase algo como un asalto o secuestro. Con este diseño se propone darle un nuevo rumbo al diseño, contemplando los problemas más importantes en la actualidad.

\section{Palabras clave}

Inseguridad; diseño industrial; asalto; mobiliario urbano; producto inteligente.

\section{Abstract}

In the national and worldwide context a lack of safety is evident in the streets. From assaults to kidnaps, these events happen frequently and the great majority of times people have no ways to notify or prevent the situation. After months of repeatedly having the same articles showing up in the newspapers, the idea of developing two smart products to combat this problem was born.

Through a market study the most important need came to light: being able to notify contacts and authorities in case of an assault or any other similar experience in the streets.

The alternatives for the product were developed after defining a design concept to guide the project. The final products were designed based in the context of a "smart city". Through 3D modeling and simulations, a set of two smart products were conceived to satisfy the main need 
that was previously defined and provide the users a tool to reduce the feeling of unease and insecurity in the streets.

Five main stages were followed to completely know and understand principal aspects of the design, such as context, aesthetics, design concept, manufacturing process, etc. Through the exploration it was possible to develop two smart products, a street product and a personal object, validated through renders, to increase the sensation of safety in the streets of Costa Rica.

\section{Keywords}

Street safety; industrial design; assault; urban mobiliary; smart product.

\section{Introducción}

En el 2020, se ha visto un incremento en asaltos, femicidios, violaciones, entre otros sucesos que ocurren en las calles. Actualmente, hay un promedio de 25 asaltos por día en el país [1]. Lo cual se ha convertido en un evento tan común que ha ido incrementando el miedo de un 82.9\% de las personas cuando transitan por las calles, según una encuesta propia aplicada a 217 personas.

Según un estudio realizado por Juan Diego García Castro y Marianela Villalobos Ulate de la Universidad de Costa Rica, en una encuesta aplicada a distintos países, se descubrió que el problema más importante de las viviendas de las personas es la inseguridad. Además, en Costa Rica se pudo observar que el mayor problema es la delincuencia [2].

Actualmente, en Costa Rica existe un aproximado de 1,336,681 mujeres y 1,240,000 hombres entre 15 y 50 años [3]. De ellos, el 60\% vive en la Gran Área Metropolitana (GAM), que es precisamente el epicentro de violaciones y asaltos en el país, como se muestra en la figura 1 [4]. En los mapas se puede observar, a la izquierda, de color rojo, el mapa de calor de violaciones y en el lado izquierdo con color azul, el mapa de calor de asaltos. Es muy utópico hablar de una ciudad donde no exista delincuencia, por lo que el producto por diseñar no pretende eliminar la

violencia en la calle. Sin embargo, se propone mantener al usuario informado sobre la situación de los alrededores para que esté seguro. 


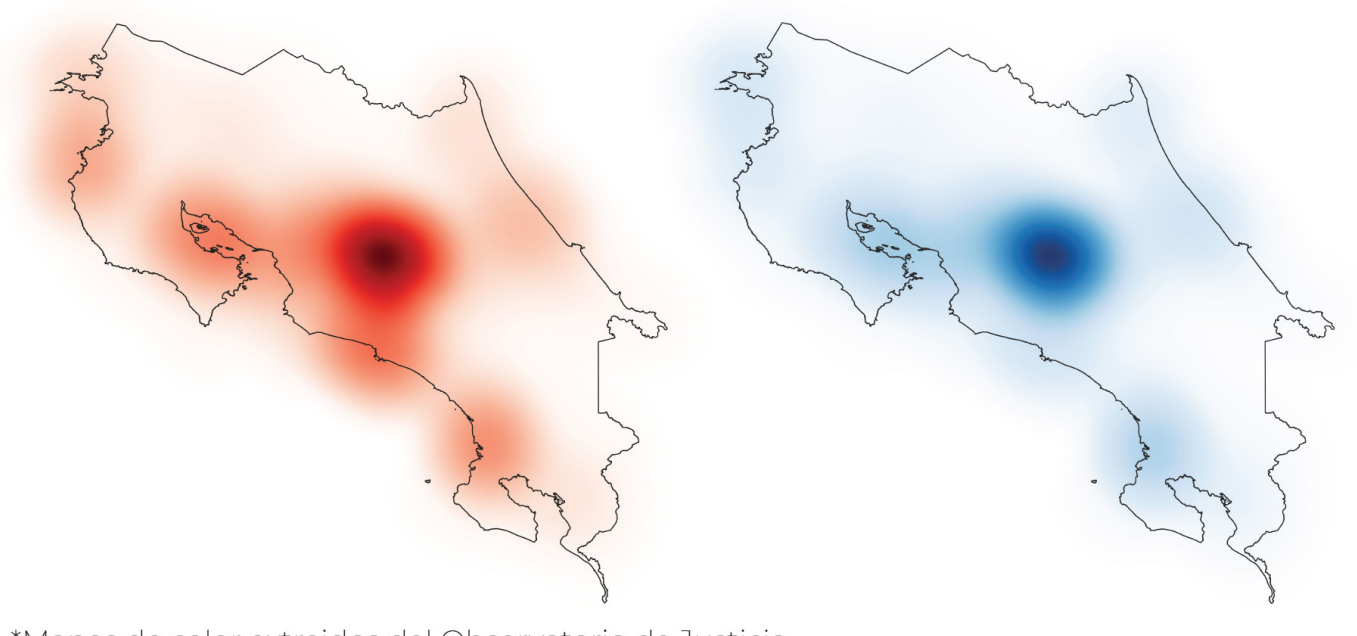

* Mapas de calor extraidos del Observatorio de Justicia

Figura 1. Mapa de Calor de Violencia en Costa Rica Fuente. Observatorio de la Justicia, 2019

Actualmente, existen productos para alertar a contactos de emergencia en caso de peligro. Los productos actuales se segmentan en tres categorías: objetos portátiles de alerta, objetos para defensa personal y aplicaciones móviles. Los objetos portátiles de alerta únicamente funcionan con conexión al celular, lo cual es una gran desventaja, ya que, si al usuario le roban el celular, dicho objeto se vuelve inútil. Esto mismo ocurre con las aplicaciones móviles.

En cuanto a los productos de autodefensa, estos son efectivos únicamente si la persona sabe cómo utilizarlos de manera correcta y sabe reaccionar en el momento, por lo que no son aptos para la mayoría. Sin embargo, estos productos no ofrecen maneras de prevenir la situación (como, por ejemplo, recomendando al usuario que no transite en ciertas calles) y se convierten en inútiles si se despegan del celular. Por esto, esos productos cumplen solamente la necesidad de alertar a seres queridos en caso de peligro, pero no permiten seguir funcionando sin el celular o continuar la comunicación con los seres queridos a quienes se alertó.

El objetivo general de la investigación es diseñar un sistema inteligente que contribuya a generar una sensación de seguridad en el usuario al caminar solo(a) en las calles. Como objetivos específicos, se propone notificar emergencias a seres queridos de manera sencilla, notificar a peatones el estado de seguridad de diferentes zonas y poder monitorear asaltos para obtener reportes precisos. Además, dado que se desarrolla en modalidad virtual, se propone generar una buena simulación que demuestre el impacto del producto, a través de un concepto amplio y trabajado como solución al problema planteado. 


\section{Metodología}

Para el desarrollo del proyecto se empleó una metodología de diseñar el objeto de adentro hacia afuera. En otras palabras, primeramente, se hizo la conceptualización de la idea para luego definir la funcionalidad y forma a partir de la misma. A continuación, se pueden observar las fases del proyecto y lo que se exploró en cada una de ellas.

\section{Conceptualización de la idea}

En este paso se exploró a fondo la problemática de la inseguridad en las calles. Pasó de ser una idea inicial a una idea con trasfondo, ya que, cuando se investigó más acerca del problema, se notó una gran importancia del mismo, debido a que, en promedio, hay 175 asaltos por semana en Costa Rica [1]. Se descubrió que el problema era de gran peso, por lo que valía la pena explorar maneras de disminuir el sentimiento de inseguridad en las calles.

Se hizo un estimado del posible mercado para descubrir si realmente sería un producto que ayudaría a muchas personas. Este cálculo, observado en la Figura 2, se hizo a partir del Método de Ratio Sucesivo y reveló que existe un mercado potencial de 1,129,144 personas. Este método toma en cuenta el universo del estudio y el porcentaje de personas que podrían adquirir el producto con base en encuestas de elaboración propia. Una vez establecidos, se aplica la fórmula mostrada en la Figura 2.

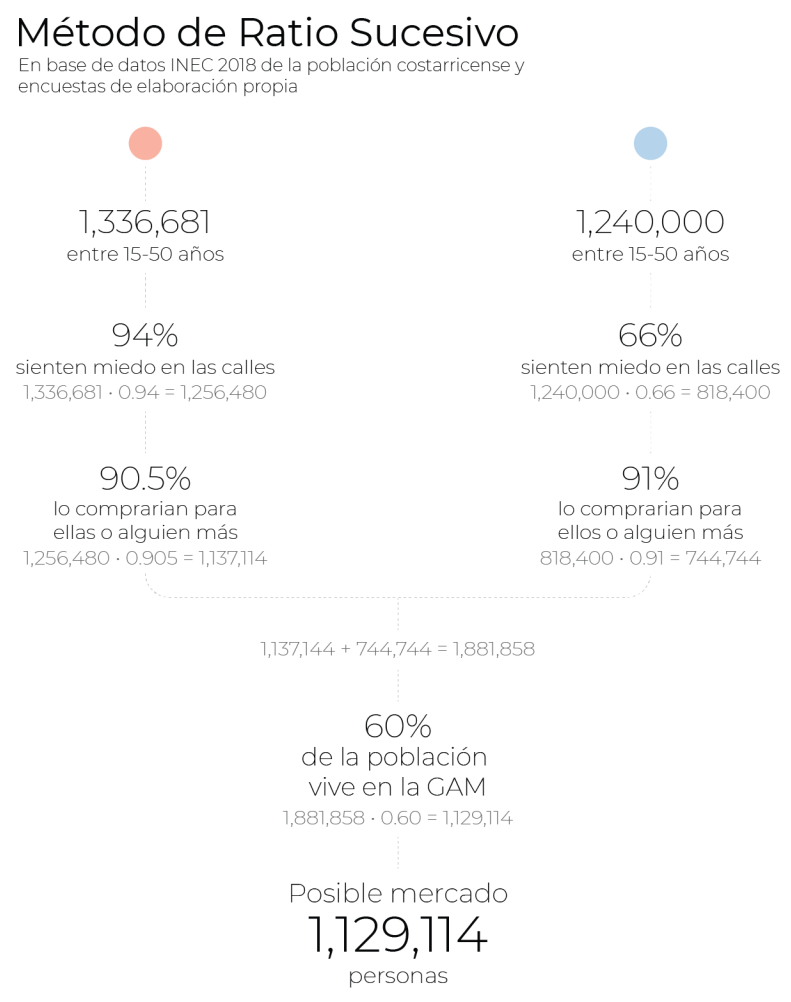

Figura 2. Cálculo de mercado por medio del Método de Ratio Sucesivo Fuente. Elaboración Propia, 2020. 
En su mayoría, se utilizaron encuestas en Instagram, con dos posibles respuestas, esto para obtener más participación por parte de las personas, ya que es la manera más sencilla para que participen en este tipo de encuestas. También se crearon entrevistas (observadas en el inicio del video [6]) para conocer más a fondo la sensación de las personas en los diferentes escenarios. Se tomó la decisión de realizar entrevistas a diferentes tipos de personas: personas nunca asaltadas y previamente asaltadas. Esto con el fin de conocer más a profundidad los efectos psicológicos de la inseguridad en las calles sobre las personas y evidenciar mejor la necesidad de resolver el problema.

De acuerdo con esto, se definieron dos usuarios principales por tomar en cuenta en la realización del proyecto. Se aplicaron entrevistas a personas que calzaban con el perfil definido de los usuarios para empatizar más con ellas. A partir de estas entrevistas, se revelaron las necesidades principales de los usuarios y se pudo delimitar un objetivo principal del producto.

Se realizó un análisis de referenciales para conocer qué productos o servicios existen que cumplan una función similar a la del producto desarrollado, esto porque es necesario conocer qué existe en el mercado y así buscar una manera para mejorar y diferenciarse de los productos existentes.

También se buscó conocer de qué manera estos productos resolvían las distintas necesidades, la interacción con los usuarios de cada uno, cómo se manejaba conceptualmente cada proyecto, materiales utilizados, entre otras características que fueron tomadas para las distintas soluciones propuestas.

\section{Definiendo la funcionalidad}

Este paso fue de suma importancia para el desarrollo del proyecto, debido a que se siguió el diseño desde "adentro hacia afuera". Se definió la funcionalidad de manera detallada a través de diagramas de funciones y diagramas de flujo, para entender de qué manera funciona el producto. Además, el análisis de las funciones permitió visualizar de manera clara las partes que serían necesarias para el producto, con el fin de contemplarlas a la hora de diseñar. En este punto, se definió que un solo producto no podría satisfacer las necesidades del usuario de manera óptima, por lo que se decidió hacer un conjunto de productos: uno de ciudad (Totem) y otro personal (Totem Mini).

Al tener una visualización de las funciones, se procedió a realizar una investigación tecnológica para tener en mente las funcionalidades posibles a nivel electrónico. También se realizaron bocetos volumétricos para empezar a darle vida al objeto, cuya función sería hacer encuestas rápidas a usuarios para verificar que se entendiera cómo funciona. 


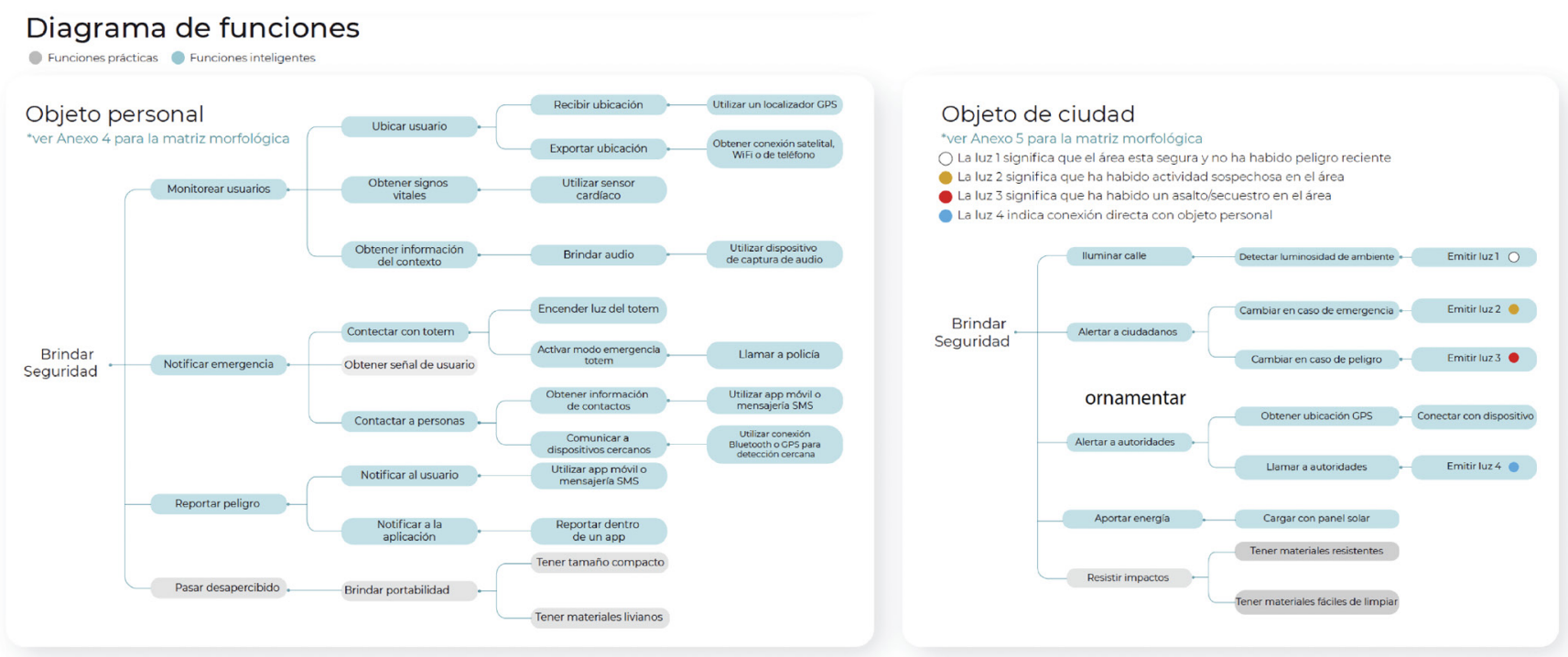

Figura 3. Diagrama de funciones de Totem y Totem Mini

Fuente. Elaboración Propia, 2020

La etapa Definiendo la funcionalidad fue crucial para el diseño de los productos porque brindó un punto de partida para el diseño. Se puede observar en la Figura 3, el diagrama de funciones y la definición del producto como sistema para Totem y el Totem Mini, en las que queda claro por qué es necesario implementar dos productos, en vez de uno solo. En esta fase se definieron flujogramas muy detallados para también tomar en cuenta la interacción con el objeto. Además, la definición de sistemas y subsistemas permitió visualizar el producto a nivel volumétrico, proporcionando una guía clara para diseñar.

\section{Definiendo la forma}

En esta etapa se definió el concepto de diseño para el producto junto con sus parámetros, objetivos generales y específicos del objeto, alcances, expectativas y "WWWWWH" (what, why, when, who, where, how), con el fin de tener un punto de partida para el diseño de los productos. Tomando en cuenta todos los parámetros del concepto de diseño establecido, se realizó un análisis perceptual a partir de un eje semántico, moodboards y matrices cromáticas. Una vez que se realizó ese estudio, se procedió a realizar varios bocetos de los productos. A partir de los bocetos generados, se realizó una matriz de toma de decisiones para evaluar cuál de las propuestas cumplía mejor con el concepto y con los requerimientos definidos en la etapa 2.

Una vez seleccionada una propuesta final, se procedió a detallarla de acuerdo con la perceptibilidad definida. Es muy importante mencionar que el diseño se realizó con base en el diagrama de funciones realizado anteriormente para tener una guía clara de los elementos que debería llevar el producto. 
En este punto comenzó un proceso iterativo de mejora de la propuesta y validarla a través del concepto de diseño y los usuarios. Después de hacer validaciones por medio de usuarios que calzaban con el público meta, se hicieron cambios al producto hasta llegar a una forma y perceptualidad final.

\section{Detallando la manufactura}

Debido a la modalidad virtual en la que se diseñó el objeto debido al COVID-19, se detalló la manufactura del producto como si fuese a realizarse en la vida real. En esta fase se exploraron los mejores materiales para utilizar y la manufactura tomando en cuenta calidad, costos y acabado. En este punto, se contemplaron en todo momento las funciones definidas previamente para poder desarrollar un producto funcional y estético con un proceso de manufactura óptimo (Figura 4).

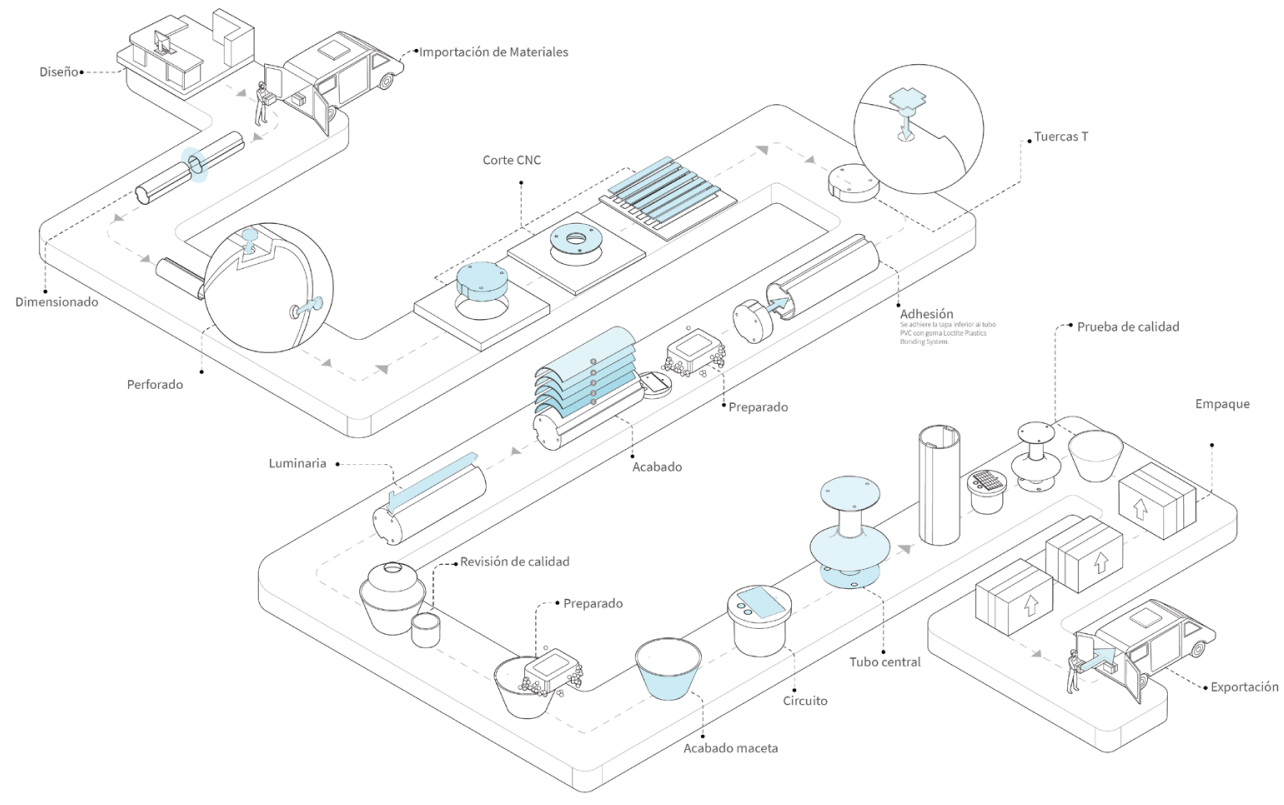

Figura 4. Proceso de Manufactura de Totem

Fuente. Elaboración Propia, 2020

También se definieron los procesos de manufactura para ambos productos, tomando en cuenta un escenario en el que los productos se fueran a producir en masa. Se realizó una investigación extensa para llegar a los materiales óptimos para los productos hablando con profesores y profesionales en el área (Figura 5). 

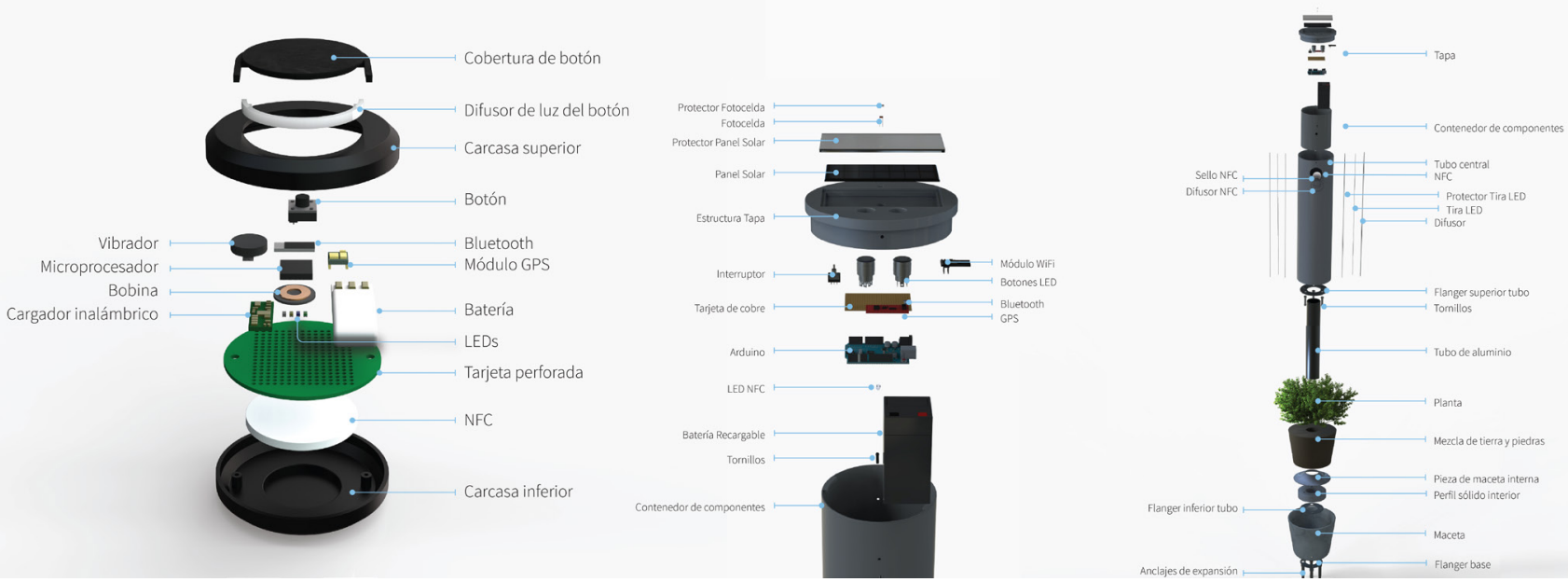

Figura 5. Exploso de productos con detalle de componentes electrónicos

Fuente. Elaboración Propia, 2020

Además, se generaron las guías de uso para indicar a los usuarios la manera en que se debe utilizar el objeto. Es importante contemplar esta parte dentro del diseño, ya que la instalación e interacción influencia grandemente el diseño del producto y puede diferenciar un buen diseño de uno excelente. Adicionalmente, se diseñó un app para configurar el Totem Mini con información del usuario, contactos de emergencia y zonas seguras. Al mismo tiempo, este app puede mostrar un mapa de calor indicando el estado de cada Totem y, por ende, el estado de seguridad de cada calle.

\section{Simulación del diseño}

Para la fase final del producto, debido a la imposibilidad de hacer validaciones de usuario por la pandemia mundial, se aplicó una serie de simulaciones del diseño para visualizarlo lo más cercano a la realidad. Se realizó una serie de renders de muy alta calidad en el software Keyshot para simular el look and feel del objeto junto con su funcionalidad. Además, se creó un video explicativo con el fin de resumir todos los aspectos definidos en la etapa 4 y que cualquier persona pudiera ver y entender el funcionamiento del producto.

\section{Discusión de resultados}

Todas las etapas fueron esenciales para un diseño integral del producto. Luego de realizar cada fase, se pudo obtener hallazgos de suma importancia para el desarrollo óptimo de los productos.

A partir de una investigación de contexto, se definieron los dos usuarios principales para el proyecto: mujeres y hombres entre 15 y 50 años, cuyo principal método de transporte es a pie y en transporte público, ya que son las personas que más experimentan asaltos. Las 
entrevistas realizadas revelaron las principales necesidades de estas personas: autodefensa, reaccionar fácilmente bajo presión, así como alertar a personas cercanas y seres queridos en caso de emergencia. Se eliminó la posibilidad de diseñar un producto de autodefensa, debido a que es un paso muy riesgoso dentro de un asalto, por lo que se centralizó el proyecto en poder reaccionar fácilmente y alertar a otras personas. Se inició pensando que el problema por resolver era la violencia en las calles. Sin embargo, con la investigación de contexto se logró descubrir las necesidades esenciales de las personas que se resumen en poder caminar por las calles sin sentirse inseguros o inseguras.

Para el diseño de Totem, se tomó en consideración un concepto de seguridad versátil por medio de los parámetros de estética, percepción, comunicación, inteligencia y configuración. Partiendo de este concepto, se podría realizar un producto que cumpliera no solamente con las necesidades "obvias" del usuario, como llamar a contactos y poder alertar, sino también las necesidades más psicológicas, como sentir seguridad emitida por la estética del producto, por ejemplo. Estas necesidades psicológicas se pudieron traducir a un elemento tangible a través de un análisis morfológico de objetos que se consideran como "seguros" (Figura 6).

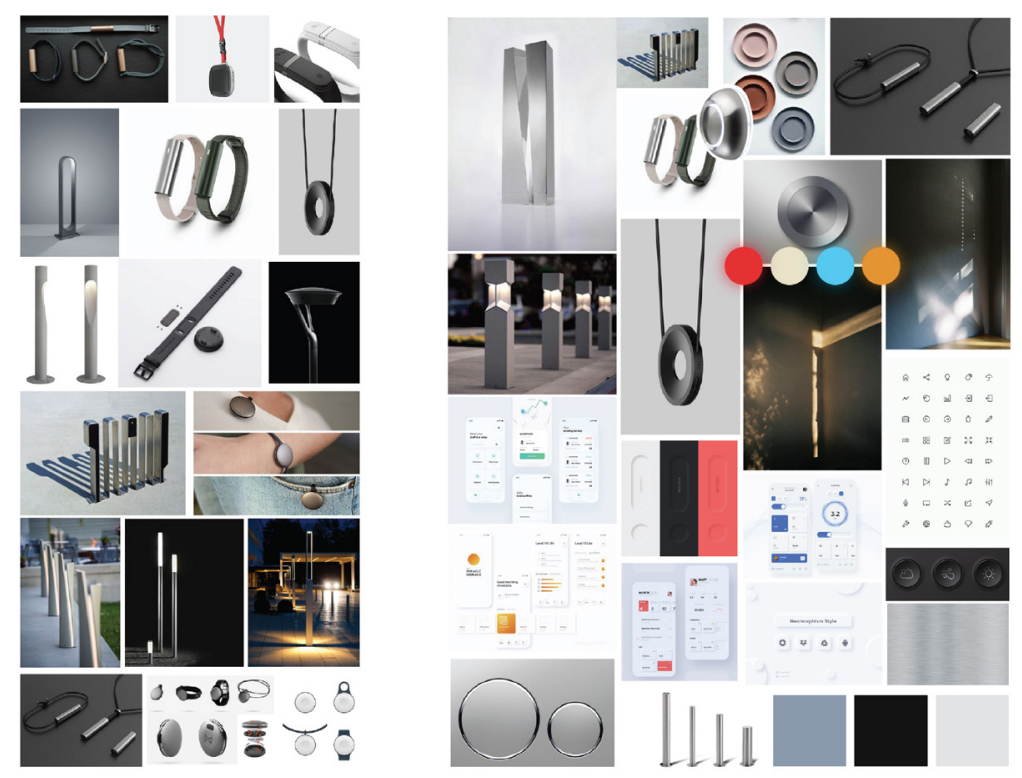

Figura 6. Frase semántica y moodboard

Fuente. Elaboración Propia, 2020

El proyecto comenzó con una población llena de miedo en su día a día (validado por encuestas realizadas). Una vez que se diseñó el proyecto, se pudo validar por medio de una encuesta realizada mediante Instagram a 127 personas que, al utilizar el producto, incrementaría el sentimiento de seguridad en las calles. Es importante reconocer que aún existe un porcentaje que no ve este cambio. Sin embargo, se necesitaría una validación más precisa y presencial para poder validar la eficacia del producto con certeza. 


\section{¿Cómo Totem resuelve la problemática?}

Es muy utópico hablar de una sociedad donde no exista maldad. Por lo tanto, Totem no plantea eliminar esto, sino alertar a los peatones para prevenir que eventos de este tipo ocurran, al ser evitados. Además, Totem no solamente funciona para las personas que tienen el Totem Mini, sino para todos los peatones que transiten en un lugar. Alimentando una base de datos con alertas generadas por usuarios de Totem mini, los Totem podrán actualizarse en tiempo real para indicar el estado de seguridad de una calle en el momento que un peatón la transita. De este modo, si un peatón ve que una ruta tiene Totems rojos o naranjas, podrá evitarla o transitarla de manera más cautelosa.

Por su parte, Totem Mini pretende, además de alimentar la base de datos, darles la posibilidad a las personas de notificar a sus seres queridos y autoridades en caso de emergencia. De este modo, si ocurre un secuestro, tendrán la ubicación real en todo momento. O si, por ejemplo, ocurre un asalto y la persona queda sin su celular, puede dirigirse a un Totem para enviar su ubicación real a usuarios de confianza que la puedan ayudar.

La planta de Totem (ver Figura 7) podrá parecer un simple elemento estético. Sin embargo, se coloca con el fin de brindar más tranquilidad [4] en el usuario, en caso de haber sido asaltado y acudir a Totem para llamar a seres queridos. De esta manera, combinado con la luz azul [6] que emite el Totem cuando detecta conexión, el usuario tendrá estímulos relajantes para ayudarle a mejorar su estado de ánimo.

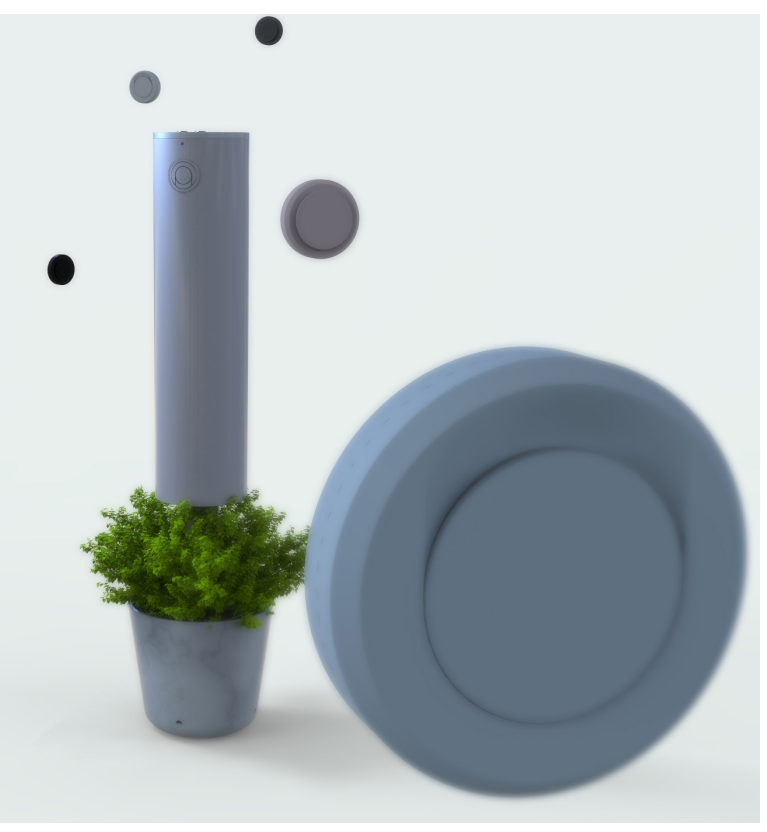

Figura 7. Simulación 3D de Totem

Fuente. Elaboración Propia, 2020 
Como se mencionó anteriormente, Totem puede comunicar a los peatones el estado de seguridad de una calle, mientras Totem Mini puede indicarle de manera directa a los usuarios a través de luces. Una luz anaranjada indica actividad sospechosa en la zona en las últimas 4 horas, notificando a los peatones que probablemente hay personas sospechosas en la zona. Una luz roja indica que hubo un asalto en las últimas 2 horas en la zona, indicando que el asaltante podría seguir cerca. Una luz azul indica conexión con el Totem Mini para darle una señal al usuario de que la conexión con el Totem Mini fue exitosa. Por último, una luz blanca, además de iluminar la calle, indica una zona segura donde no ha habido reportes recientes.

Es importante mencionar que se requiere de un Totem Mini para interactuar con el Totem, con el fin de prevenir reportes erróneos y procurar reportes auténticos. Además, se requiere de un Totem Mini para que el Totem obtenga una base de datos de contactos a quienes llamar.

\section{Hallazgos técnicos}

Al definir la manufactura de los objetos, es importante mencionar la importancia de investigar todas las opciones disponibles de material. Al inicio se planteó construir la estructura primaria del objeto principal de acero inoxidable. Sin embargo, al plantear este material de manera precipitada, no se tomaron en cuenta factores como el peso, el costo del material y el costo de producción. Luego de hacer la investigación de la etapa 4, se decidió reemplazar el material por PVC con dióxido de titanio, que resiste impactos y al clima. Adicional al PVC, se definió el uso de HDPE para piezas secundarias del Totem y sí se mantuvo el uso de aluminio, pero únicamente para la barra central que estaría en contacto con la planta. De esta manera, se preservaría la resistencia mientras se reducen los costos y se simplifican los procesos de manufactura.

Además, el uso de PVC, que es mucho más liviano, permite una instalación más sencilla debido al peso reducido. La instalación del Totem se puede observar en la Figura 8.

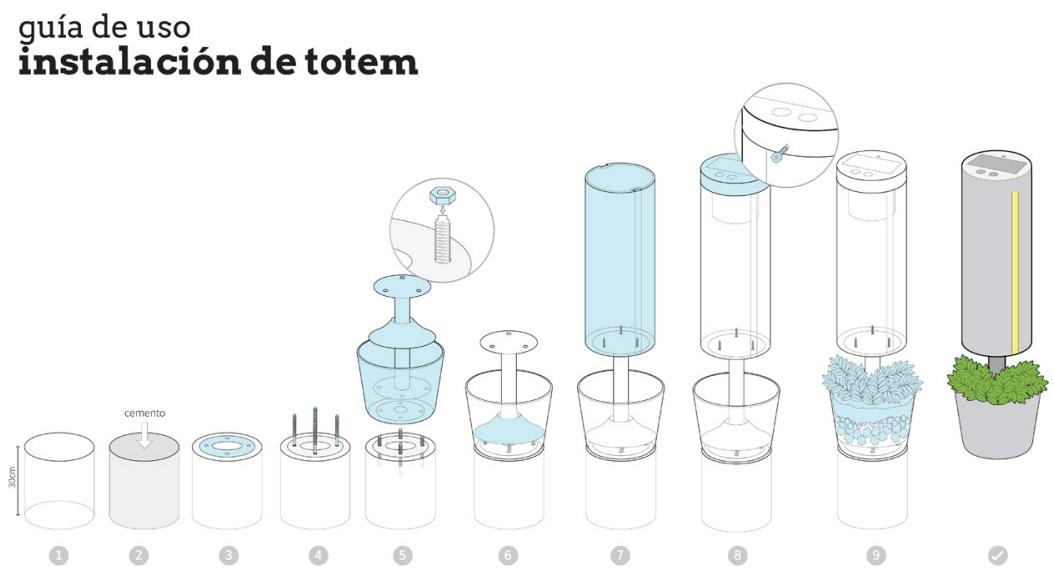

Figura 8. Manual de instalación para Totem

Fuente. Elaboración Propia, 2020 
Para Totem Mini se definió el uso de ABS por su alta resistencia a impactos, ya que, al ser un objeto portátil, probablemente sufra impactos. Para los difusores de luz, se seleccionaron láminas de PP (Polipropileno) debido a la difusión efectiva de la luz y su resistencia.

Un factor interesante en cuanto a la investigación morfológica que debía tener Totem Mini fue la posibilidad de disimular el objeto para que, en caso de ser asaltado, el asaltante no lo robe. Por eso, Totem Mini cuenta con piezas adicionales de hule de silicón para adaptar Totem Mini en forma de llavero, collar, pulsera, o bien andarlo suelto para llevarlo en el bolsillo (Ver Figura 9). También, se propuso una textura mate para prevenir que, al emitir una luz de alerta, llamara mucho la atención. Otro detalle importante de Totem Mini es la curvatura leve que lleva. Esto no solo permite una mejor visualización de la luz de alerta, sino que también mejora la visibilidad de la luz, en caso de estar en un ambiente muy claro, generando una leve sombra interna para que la luz pueda reflejarse.

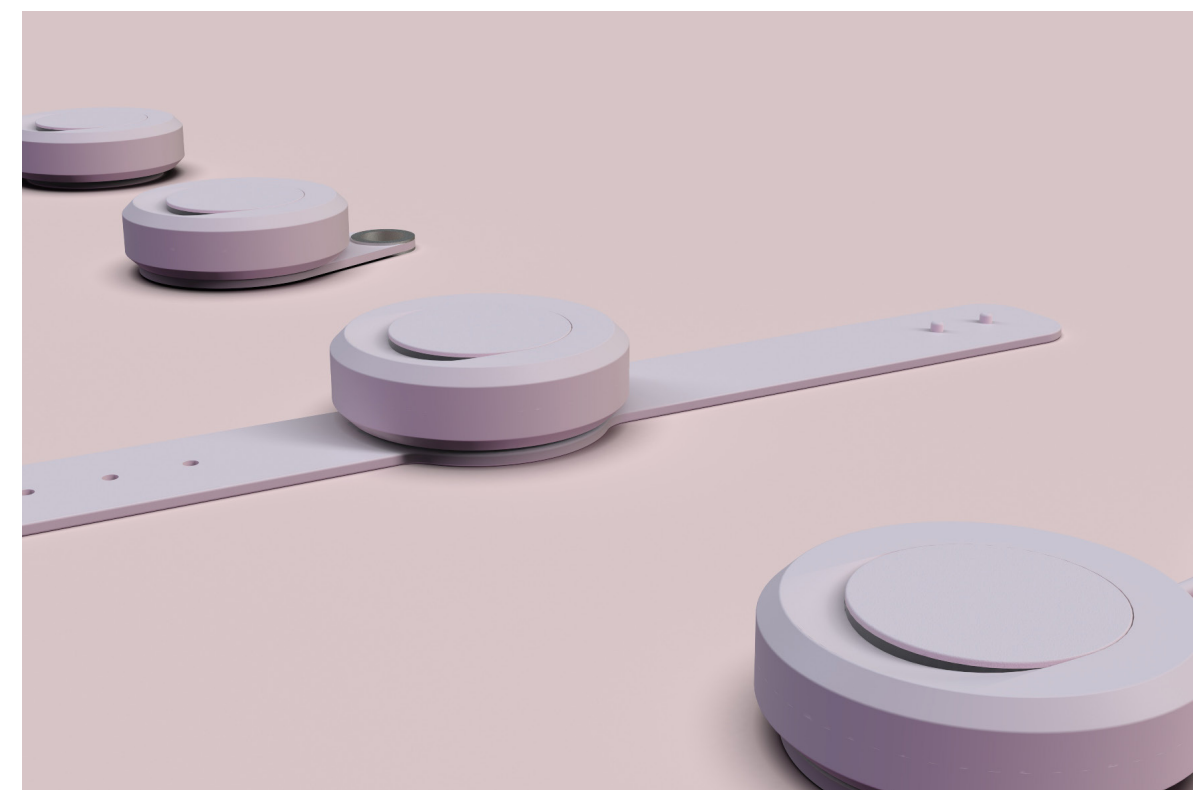

Figura 9. Simulación 3D de Totem Mini

Fuente. Elaboración Propia, 2020.

\section{Uso del Totem Mini y Totem}

A continuación, se puede observar una parte del resultado final, que se profundiza en el video citado [6]. 


\section{guía de uso totem mini}

¿qué viene en la caja?

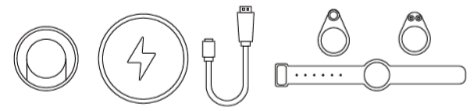

Señalización

El Totem Mini notificará al usuario en 4 ocasiones po medio de una vibración y una luz discreta. Los tipos de luz son los siguientes:

\section{Instalación inicial}

(1) Descargar el app de Totem Mini al celular

2 Ingresar datos personales (contactos de emergencia, zonas verdes, preferencia de uso) Sincronizar Totem Mini con el app por medio
de Bluetooth

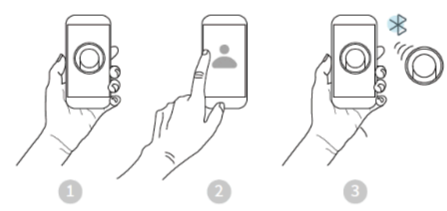

Poner accesorio

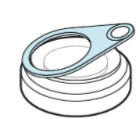

Insertar esquina de accesorio a pieza de entrada

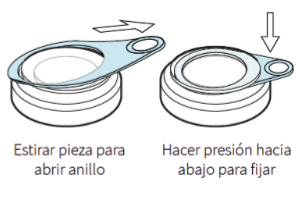

abrir anillo

Remover accesorio

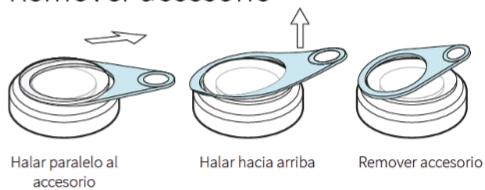

En caso de emergencia Presionar el botón por más de 1

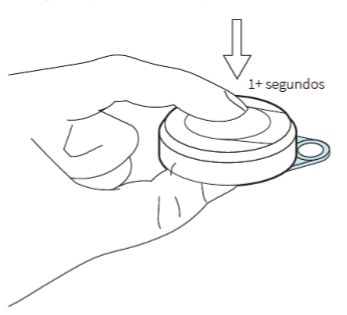
Presionar el botón más de 2 veces rápidamente para indicar emergencia

En caso de asalto Dirigirse al bolardo más cercano y (-) llamar a contactos - llamar a autoridades

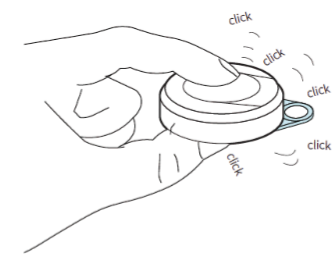

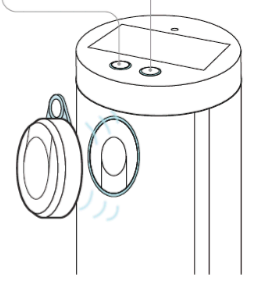
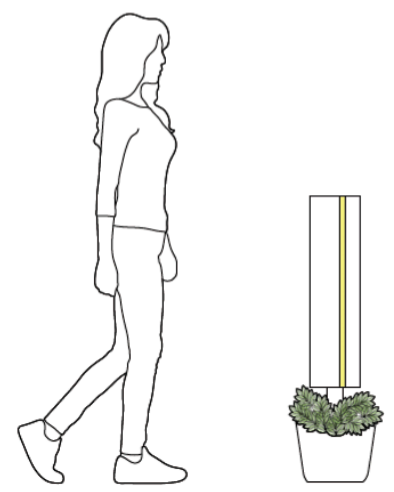

(2) llamar a contactos (-) llamar a autoridades
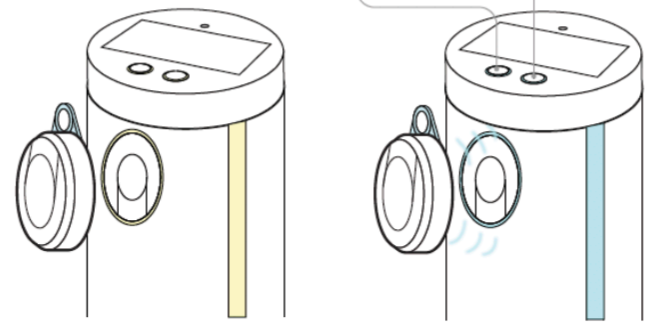
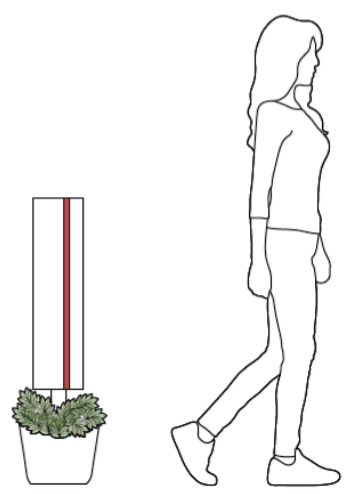

Figura 10. Uso del Totem mini y Totem.

Fuente. Elaboración propia.

\section{Conclusiones}

Este proyecto y sus diferentes pasos revelaron, principalmente, que se debe indagar más allá de la superficie para obtener un diseño realmente efectivo. El hecho de analizar el problema a fondo desde el inicio del proyecto permitió generar un objetivo claro para el desarrollo del mismo. 
Desarrollar el producto de manera remota permitió concentrarse en las funciones principales del objeto. Sin embargo, como aspectos por mejorar, se considera que, para aumentar la inteligencia del Totem, se podría implementar un sistema de riego automatizado para las plantas, con el fin de poder seleccionar una variedad de planta que requiera riego controlado y pueda aportar aún más al medio ambiente.

Este proyecto demuestra cómo es posible atacar diferentes problemáticas desde puntos de vista diferentes. Una persona podría pensar que la solución más simple para la inseguridad en las calles es posiblemente aumentar la cantidad de policías en las calles o atrapar a los asaltantes. Sin embargo, esa idea es sumamente utópica e irreal. En su lugar, con este proyecto se pudo buscar un nuevo punto de vista, mezclando la realidad con una situación de seguridad en las calles un poco mejor que la actual.

El resultado de las etapas utilizadas para el desarrollo del producto fue un diseño integral y funcional, dirigido a los usuarios para satisfacer su necesidad de sentir más seguridad caminando por las calles. Además, con este proyecto se pudo observar cómo los productos y la electrónica viven cada día más en sintonía para crear productos inteligentes. En la modernidad ese podría ser el nuevo rumbo del diseño de productos.

\section{Referencias}

[1] Observatorio de la Violencia. Mapas. Disponible: http://observatorio.mj.go.cr/recursos/ mapas.

[2] J. D. García Castro and M. Villalobos Ulate, "¿Vivimos en un lugar seguro? Percepción de criminalidad en la zona de Occidente," Revista Pensamiento Actual, 2016. Disponible: https://revistas.ucr.ac.cr/index.php/pensamiento-actual/article/view/27441.

[3] INEC - Costa Rica "Costa rica en cifras". Disponible: https://www.inec.cr/sites/default/files/ documetos-biblioteca-virtual/recostaricaencifras2018.pdf

[4] OIJ - Costa Rica. Estadísticas Policiales del OIJ. Disponible: https://pjenlinea3.poderjudicial.go.cr/estadisticasoij/.

[5] Charles A. Lewis, The meaning of plants in our lives, Green Nature Human Nature, 1996. Disponible: https://books.google.es/books?hl=en\&lr=\&id=ymAymjOG3QQC\&oi=fnd\&pg= PP13\&dq=plants+relax+people\&ots=0G0Nmhx8oB\&sig=ds8ks6Z940KSQIONiE-j7U_G_ $14 \# v=$ onepage \&q=plants\%20relax\%20people\&f=false

[6] Corella J., González M., Totem: diseño de productos para ciudades inteligentes Disponible: https://www.youtube.com/watch?v=WQKOVF6v8Vo

[7] F. Birren, Color Psychology and Color Therapy; A Factual Study of the Influence of Color on Human Life. Hauraki Publishing, 2016. 